\title{
Análise Descritiva por Ordenação: aplicação na caracterização sensorial de biscoitos laminados salgados
}

\author{
Ranking Descriptive Analysis: application in the \\ sensory characterization of cracker biscuits
}

\section{Autores | Authors}

Lorena CARNELOCCE

Universidade Estadual de Londrina (UEL) Departamento de Ciência e Tecnologia de Alimentos Londrina/PR - Brasil e-mail: Iorena.araujoc@gmail.com

Neusa Fátima SEIBEL

Universidade Tecnológica Federal do Paraná (UTFPR)

Departamento Tecnologia de Alimentos Londrina/PR - Brasil e-mail: neusaseibel@utfpr.edu.br

Sandra Helena PRUDENCIO

Universidade Estadual de Londrina (UEL) Departamento de Ciência e Tecnologia de Alimentos Londrina/PR - Brasil e-mail: sandrah@uel.br

\Marta de Toledo BENASSI

Universidade Estadual de Londrina (UEL) Departamento de Ciência e Tecnologia de Alimentos

Rod. Celso Garcia Cid, PR 445, Km 380 Campus Universitário Caixa Postal: 6001 CEP: 86051-990 Londrina/PR - Brasil e-mail:martatb@uel.br

$\triangle$ Autor Correspondente / Corresponding Author

Recebido / Received: 18/04/2011 Aprovado / Approved: 07/07/2012

Publicado / Published: dez./2012

\section{Resumo}

A Análise Descritiva por Ordenação (ADO) foi aplicada na caracterização de biscoitos cream cracker e água e sal comerciais, empregando julgadores pré-selecionados, mas sem experiência prévia em análise sensorial. O método compreendeu as etapas de levantamento e conceituação dos atributos, treinamento qualitativo e análise final, utilizando o procedimento de ordenação. Para verificação da eficiência na discriminação das amostras, o procedimento de ordenação foi comparado ao uso de escala não estruturada. Dados de caracterização instrumental de textura e cor foram comparados aos obtidos na descrição sensorial dos biscoitos. Análise Procrustes Generalizada foi utilizada na avaliação dos dados de ordenação ou escala, e os resultados da ordenação foram também avaliados por Teste de Friedman. A eficiência da equipe e a configuração das amostras foram similares no procedimento de ordenação e com o uso de escala. Após um treinamento qualitativo e uma avaliação inicial das amostras por um procedimento de ordenação, os julgadores não apresentaram divergência de comportamento, mesmo empregando um procedimento mais difícil (uso de escala). Cor e aroma de assado e sabor tostado, aroma de manteiga e sabor amanteigado, crocância (manual e na boca) e uniformidade da cor foram os atributos mais importantes na discriminação. A ADO permitiu a caracterização e a discriminação de modo concordante com a composição indicada no rótulo dos produtos e os resultados instrumentais de textura e cor.

Palavras-chave: Cream cracker; Textura; Friedman; Análise Procrustes Generalizada.

\section{Summary}

Ranking Descriptive Analysis (RDA) was applied to the characterization of commercial cream cracker and water and salt biscuits, using pre-selected assessors with no previous experience in sensory analysis. The method had the following steps: development of attributes, glossary and references, qualitative training and final analysis using the ranking procedure. To check the efficiency in discriminating the samples, the ranking procedure was compared to the use of a non-structured scale. Instrumental color and texture data were compared with the characterization obtained from the sensory description of the cracker biscuits. The Generalized Procrustes Analysis was applied to the data analysis (ranking or scale), and the ranking results were also evaluated by the Friedman test. The efficiency of the panel and the configuration of the samples were similar in both the ranking procedure and using the scale. After a qualitative training and an initial evaluation of the samples by a ranking procedure, the assessors showed similar behaviour, even when using a more difficult procedure (use of scale). Roasted colour and aroma, toasted flavour, butter aroma, butter flavour, crispness (manual and in the mouth) and uniformity of colour were the most important attributes for discrimination. The RDA allowed a characterization and discrimination consistent with the composition specified on the labels of the products and with their instrumental texture and colour results.

Key words: Cracker biscuits; Texture; Friedman; Generalized Procrustes Analysis. 


\section{Introdução}

A metodologia de Análise Descritiva por Ordenação (ADO) foi desenvolvida por Richter et al. (2010) como uma alternativa à análise descritiva tradicional, a qual requer treinamento longo e caro dos julgadores para fornecer resultados confiáveis e consistentes (STONE e SIDEL, 1998). A ADO foi comparada com dois métodos descritivos - Análise Descritiva Quantitativa (ADQ) e Perfil Livre, usando-se pudins de chocolate como amostras. Os julgadores pré-selecionados desenvolveram uma lista de descritores com seus respectivos conceitos e, após receberem treinamento qualitativo, avaliaram as amostras pelo procedimento de ordenação. A técnica permitiu discriminação das amostras estudadas com eficiência semelhante às descritivas e obteve-se maior consenso da equipe e variância residual mais baixa (RICHTER et al., 2010). O consenso foi atribuído à facilidade do procedimento de ordenação, já mencionada na literatura (BARYLKO-PIKIELNA et al., 2004; DELARUE e SIEFFERMANN, 2004; KIM e O'MAHONY, 1998; LEE e O'MAHONY, 2005; LIEM et al., 2004; RODRIGUE et al., 2000).

Desse modo, a ADO oferece algumas vantagens em relação à $A D Q$, como dispensar o treinamento para a padronização de extremos de escala e a seleção dos julgadores e possíveis retreinamentos, diminuindo o tempo e o custo na descrição de um produto, na medida em que necessita de um menor número de sessões e menor quantidade de amostra. No entanto, para avaliar a eficiência e validar a metodologia, Richter et al. (2010) enfatizaram que a técnica deveria ser empregada na análise de matrizes alimentares mais complexas e de características sensoriais diferenciadas (número e natureza dos atributos), o treinamento qualitativo deveria ser expandido e a repetibilidade do método deveria ser verificada.

Em estudo recente, Carnelocce (2011) verificou a eficiência e a repetibilidade da ADO na caracterização de cinco refrescos. A equipe apresentou consenso e repetibilidade na descrição, e os refrescos foram caracterizados e discriminados de forma concordante com a formulação e o perfil físico-químico de cada produto. No entanto, uma vez que os julgadores são forçados a ordenar mesmo que não observem diferença entre atributos, o autor questionou se o uso do procedimento de ordenação poderia implicar numa menor capacidade de discriminação do que a obtida empregando escala - usual em métodos descritivos - e sugeriu estudos posteriores para essa comparação.

A textura e a cor são propriedades sensoriais de grande relevância na aceitabilidade dos alimentos. A análise sensorial dessas características necessita de padronização cuidadosa, pois está sujeita às condições do ambiente, como iluminação e umidade relativa, e à acuidade do julgador. A medida instrumental apresenta resultados rápidos, exatos e precisos que, quando bem correlacionados com dados sensoriais, facilitam a descrição do produto (DURÁN, 1999; RICHTER et al., 2010; SCHIFFERSTEIN, 2006). A percepção da textura dos alimentos se dá pela combinação dos sentidos sensoriais (VARELA et al., 2009), o que dificulta a avaliação sensorial dessa característica, uma vez que o entendimento dos atributos pode ser diferente dependendo dos julgadores empregados (CHAUVIN et al., 2008; CHEN et al., 2005). Portanto, para uma correlação entre resultados sensoriais e instrumentais de textura, é necessário escolher procedimentos de medidas de maneira a garantir que a técnica instrumental reproduza a descrição sensorial (BOURNE, 1978; DURÁN, 1999).

O objetivo deste trabalho foi verificar a eficiência da Análise Descritiva por Ordenação na descrição de uma matriz complexa, biscoitos laminados salgados, além de comparar o uso de ordenação e escala não estruturada na discriminação das amostras estudadas.

\section{Material e métodos}

\subsection{Material}

Foram utilizados quatro produtos dos tipos biscoitos água e sal, e cream cracker comerciais, que apresentavam descritas nos rótulos diferenças na composição quanto ao teor de gordura e sódio (Tabela 1). Os produtos continham como ingredientes: farinha de trigo fortificada com ferro e ácido fólico, gordura vegetal, amido, sal, extrato de malte, açúcar invertido, açúcar, fermento biológico, bicarbonato de sódio e acidulante ácido láctico. As amostras utilizadas, de cada produto, foram do mesmo lote de fabricação.

\subsection{Análise instrumental de cor e textura}

A avaliação de cor foi realizada em nove repetições, empregando-se colorímetro Chroma Meter CR400 (Konica Minolta, Japão), com geometria $45 / 0$ e iluminante $D_{65}$. Obtiveram-se os valores de $L^{*}$ (luminosidade), $a^{*}$ (componente vermelho-verde) e $b^{*}$ (componente amarelo-azul), e foi calculada a tonalidade cromática $\left(\mathrm{H}^{*}=\operatorname{arctg}\left(\mathrm{b}^{*} / \mathrm{a}^{*}\right)\right.$.

Tabela 1. Características dos biscoitos descritas nos rótulos.

\begin{tabular}{|c|c|c|c|c|}
\hline Produto & Marca & Tipo & $\begin{array}{c}\text { Teor de } \\
\text { gordura (\%) }\end{array}$ & $\begin{array}{l}\text { Teor de sódio } \\
\left(\mathrm{mg} .100 \mathrm{~g}^{-1}\right)\end{array}$ \\
\hline A & $X$ & $\begin{array}{c}\text { Cream } \\
\text { cracker light }\end{array}$ & 1,9 & 600 \\
\hline B & $x$ & Água e sal & 3,5 & 1057 \\
\hline C & Y & Água e sal & 3,4 & 783 \\
\hline D & Z & Água e sal & 4,0 & 673 \\
\hline
\end{tabular}


Para as análises de textura, foram adaptadas as condições propostas por Laguna et al. (2010), utilizando um texturômetro TAX-T2 (Stable Micro Systems, Inglaterra). Foram realizadas dez repetições por amostra e a força máxima (N) foi medida para cada teste.

A força de penetração foi avaliada para estimar de forma global a textura (dureza, crocância e mastigabilidade) (SALVADOR et al., 2009). Utilizou-se um sensor (probe) de $6,0 \mathrm{~mm}$ de diâmetro cilíndrico de aço $(\mathrm{P} / 6)$, força de $0,05 \mathrm{~N}$ e velocidade do teste $5,0 \mathrm{~mm} . \mathrm{s}^{-1}$. Os biscoitos foram apoiados num suporte com perfuração circular colocado em posição invertida e o sensor penetrou 10,0 mm da altura inicial.

Foi medida a força de compressão para avaliar a dureza. Dois biscoitos foram comprimidos 3,0 mm da altura inicial usando sensor de $75 \mathrm{~mm}$ de diâmetro chapa de alumínio (P/75), com força de 0,07 N e velocidade do teste 2,0 mm.s $\mathrm{s}^{-1}$.

A força de ruptura foi usada para avaliar a crocância. Biscoitos foram quebrados utilizando sensor de três pontos de apoio (Three-point Bend Rig) (A/3PB), com força de 0,05 N, velocidade do teste 1,0 mm.s $\mathrm{s}^{-1} \mathrm{e}$ distância percorrida pelo sensor de $6,0 \mathrm{~mm}$.

Os experimentos de cor e textura foram conduzidos conforme delineamento inteiramente ao acaso. Os resultados foram submetidos à Análise de Variância (ANOVA) e teste de comparação de médias (Tukey, $p \leq 0,05)$ (STATSOFT, 2001).

\subsection{Análise Descritiva por Ordenação}

\subsubsection{Recrutamento e pré-seleção dos julgadores}

Os julgadores, sem experiência prévia em testes sensoriais descritivos, foram recrutados com base na disponibilidade, tendo sido informados acerca dos produtos e procedimentos dos testes, conforme descrito no projeto cadastrado no Sistema Nacional de Ética em Pesquisa (Certificado de Apresentação para Apreciação Ética n. 0143.0.268.000-09).

Os julgadores participaram de uma pré-seleção, na qual se avaliou o desempenho em testes de ordenação de intensidade de dureza e de cor (MEILGAARD et al., 1999).

Para ordenação de intensidade de dureza, foram empregadas amostras (paçoca, biscoito cream cracker e torrada tipo canapé) selecionadas pelas diferenças na força de compressão (item 2.2). Foram utilizadas para a análise duas unidades de torrada ou biscoito, e uma unidade de paçoca, as quais foram comprimidas até $2,0 \mathrm{~mm}$ da altura inicial. A paçoca possuía menor intensidade de dureza $(15,28 \mathrm{~N})$ e a torrada, a maior $(161,15 \mathrm{~N})$ (Tabela 2).

Para discriminação de intensidade de cor, foram empregados quatro cafés conilon com diferentes graus de torra. O café 1 tinha cor mais clara, apresentando maior luminosidade e tonalidade cromática (marrom amarelado) e o café 4, o outro extremo, era mais escuro e marrom avermelhado (Tabela 3).

Foi solicitado a cada candidato que ordenasse de forma crescente a intensidade de dureza e cor das amostras. Tendo em vista que a equipe não tinha experiência com uso de escala, foi solicitado que, após a ordenação, o julgador marcasse numa escala não estruturada a intensidade de cada atributo. Assim, essa etapa também serviu para familiarização do julgador com os procedimentos a serem empregados.

Considerou-se, como critério de seleção, que os julgadores apresentassem acima de 50\% de acerto na ordenação. Todos os candidatos foram aprovados, uma vez que apresentaram a capacidade de discriminação requerida.

\subsubsection{Desenvolvimento da terminologia descritiva e treinamento qualitativo}

A análise foi conduzida conforme Richter et al. (2010). O desenvolvimento da terminologia descritiva foi feito com 30 julgadores pré-selecionados, utilizando-se o Método Rede (MOSKOWITZ, 1983). Para uso do procedimento de ordenação com um critério descritivo, é sugerido o uso de, no mínimo, 15 julgadores (ISO, 2006), sendo que Richter et al. (2010) utilizaram 21 julgadores no desenvolvimento do método. Considerando-se o uso de matriz complexa, optou-se por trabalhar com um número inicial de julgadores mais alto. Foram realizadas duas sessões, nas quais as amostras foram apresentadas

Tabela 2. Características dos produtos usados no teste de ordenação de intensidade de dureza para pré-seleção de julgadores.

\section{Produto Espessura $(\mathrm{cm})^{*} \quad$ Dureza $(\mathrm{N})^{* *}$}

\begin{tabular}{llr} 
Paçoca & 1,4 & $15,3 \pm 1,1^{\mathrm{c}}$ \\
Biscoito cream cracker & 0,5 & $120,5 \pm 1,3^{\mathrm{b}}$ \\
Torrada canapé & 0,7 & $161,2 \pm 5,7^{\mathrm{a}}$ \\
\hline
\end{tabular}

*Espessura/unidade. ${ }^{*}$ Média de três repetições \pm desvio padrão. Letras diferentes na mesma coluna indicam diferença significativa $(p \leq 0,05)$

Tabela 3. Características dos cafés usados no teste de ordenação de intensidade de cor para pré-seleção de julgadores.

\begin{tabular}{ccc} 
Café & $\mathbf{L}^{*}$ & $\mathbf{H}^{*}$ \\
1 & $33,2 \pm 0,4^{\mathrm{a}}$ & $62,9 \pm 0,4^{\mathrm{a}}$ \\
2 & $26,9 \pm 0,5^{\mathrm{b}}$ & $61,5 \pm 0,6^{\mathrm{a}}$ \\
3 & $20,7 \pm 0,7^{\mathrm{c}}$ & $56,9 \pm 0,4^{\mathrm{b}}$ \\
4 & $14,3 \pm 0,6^{\mathrm{d}}$ & $52,6 \pm 0,8^{\mathrm{c}}$ \\
\hline
\end{tabular}

${ }^{*}$ Média de seis repetições \pm desvio padrão. Letras diferentes na mesma coluna indicam diferença significativa $(p \leq 0,05)$. 
aos pares ( $A$ e C; B e D), e foi solicitado aos julgadores que comparassem o par de amostras e descrevessem similaridades e diferenças. Para facilitar o procedimento e levantar maior número de atributos, foi anexado um protocolo de avaliação, pelo qual os julgadores foram instruídos a observar a aparência, o aroma, a textura e o sabor das amostras. Solicitou-se que quebrassem um biscoito na mão e outro na boca, verificando o som e a dificuldade para quebra e mastigação, e as sensações durante mastigação e após engolir.

Após cada julgador ter gerado seus próprios termos descritivos, foram conduzidas duas sessões de discussão em grupo para selecionar os termos mais citados, agrupar os semelhantes e desenvolver uma lista de descritores consensual e um glossário (Tabela 4).

Para compreensão do significado de cada atributo, foram realizadas três sessões de treinamento qualitativo, empregando-se amostras de referências propostas pela equipe. Para os descritores considerados mais complexos pelos julgadores (uniformidade da cor, aroma e sabor característico de biscoito água e sal, aroma de assado, crocância manual e na boca), foram utilizadas referências representando diferentes intensidades (Tabela 4).

Foi realizada uma sessão final para verificar o consenso dos julgadores quanto à definição dos descritores e à ficha de avaliação. Após adequações, o protocolo de instruções foi anexado, juntamente com o glossário, nas cabines em todas as provas, para facilitar e padronizar a forma de manipular as amostras durante as avaliações.

\subsubsection{Análise Descritiva por Ordenação e uso de escala}

As análises foram realizadas em cabines individuais, com luz branca. Três unidades de biscoito de cada amostra foram servidas em pratos plásticos, codificados com três dígitos. A ordem de apresentação foi aleatorizada para cada julgador. Os julgadores lavaram suas bocas com água mineral entre as amostras.

Uma sessão foi realizada para a avaliação das amostras na Análise Descritiva por Ordenação. As amostras foram apresentadas simultaneamente e foi

Tabela 4. Definições dos descritores sensoriais e amostras de referência

\begin{tabular}{ll}
\multicolumn{1}{c}{ Descritores } & \multicolumn{1}{c}{ Definição } \\
Cor de assado & $\begin{array}{l}\text { Cor associada ao processo de assamento dos } \\
\text { biscoitos. }\end{array}$ \\
Uniformidade da cor & $\begin{array}{l}\text { Homogeneidade da cor na superfície do biscoito } \\
\text { (sem verificar possíveis presenças de bolhas na } \\
\text { superfície) }\end{array}$ \\
Aroma de manteiga & $\begin{array}{l}\text { Aroma associado à presença de manteiga. } \\
\text { Aroma característico de } \\
\text { biscoito água e sal }\end{array}$
\end{tabular}

Aroma associado ao processo de assamento dos biscoitos.

Crocância manual Força requerida para quebrar a biscoito ao meio usando as mãos e som produzido.

Crocância na boca

Mastigabilidade

Pegajosidade

Sabor Amanteigado

Sabor característico de biscoito água e sal

Sabor de tostado
Força para quebrar a biscoito com os dentes incisivos e som produzido.

Tempo necessário para mastigar o biscoito a uma velocidade constante até estar pronto para ser engolido.

Associada à aderência do biscoito na boca durante a mastigação, dando a impressão de um filme que forma na boca e sai com o tempo. Sabor associado à presença de manteiga Sabor tradicional de biscoito água e sal, associado à presença de sal.

Sabor associado ao processo de assamento dos biscoitos, relacionado ao sabor residual amargo.

\section{Referências}

Muito: Biscoito cream cracker Todeschini

Pouco: Biscoito cream cracker Todeschini.

Muito: Biscoito água e sal Itamaraty

Intenso: Biscoito cream cracker manteiga Marilan

Fraco: Biscoito água e sal Marilan

Intenso: Biscoito água e sal Mabel

Fraco: Biscoito água e sal Carrefour Intenso: Biscoito cream cracker Todeschini

Pouco: biscoito salgado aperitivo Bauducco mantido em $90,2 \%$ UR por 4 horas a $18^{\circ} \mathrm{C}$

Muito: biscoito salgado aperitivo Bauducco Pouco: biscoito salgado aperitivo Bauducco mantido em $90,2 \%$ UR por 4 horas a $18^{\circ} \mathrm{C}$

Muito: biscoito salgado aperitivo Bauducco Muito: Biscoito água e sal Parati

Muito: Biscoito de polvilho tradicional Kapella

Intenso: Biscoito cream cracker manteiga Marilan Pouco: Biscoito água e sal Itamaraty

Muito: Biscoito água e sal Duchen

Muito: Biscoito água e sal Todeschini 
Análise Descritiva por Ordenação: aplicação na caracterização sensorial de biscoitos laminados salgados CARNELOCCE, L. et al.

solicitado ao julgador que as ordenasse em ordem crescente de intensidade para cada um dos atributos. Outra sessão foi realizada para a avaliação das amostras empregando-se, para cada atributo, uma escala não estruturada de $9 \mathrm{~cm}$, ancorada nos extremos com termos de intensidade. As amostras foram apresentadas sequencialmente e foi solicitado aos julgadores que indicassem o local na escala em que a amostra estava representada.

Os resultados da ordenação e da escala foram avaliados pela Análise Procustes Generalizada (APG) utilizando-se o programa Senstools versão 2.3.28 (OP\&P Product Research, 1998), para comparação da configuração das amostras e verificação da eficiência e do consenso da equipe. Os dados da ADO foram também avaliados por teste de Friedman (NEWELL e MacFARLANE, 1987) para verificar diferenças entre amostras para cada atributo, considerando-se quatro amostras e 30 julgadores (valor crítico: 26, $p \leq 0,05$ ).

\section{Resultados e discussão}

\subsection{Caracterização instrumental de textura e cor}

Os biscoitos apresentaram diferentes perfis de textura e cor (Figura 1, Tabela 5). Deve-se considerar, no entanto, a grande variabilidade das amostras, a qual pode ser constatada pelos valores do coeficiente de variação, notadamente nas determinações de força de penetração e ruptura (CVs de 12 a 23).

Os biscoitos não apresentaram diferenças em relação à força de penetração, a qual caracteriza de forma global a textura dos produtos. Apesar das diferenças na composição, as amostras diferiram pouco na textura, indicando a necessidade de avaliar parâmetros mais específicos (crocância e dureza) para correlação com os resultados sensoriais. A amostra $\mathrm{D}$, contendo o maior teor de gordura (Tabela 1), foi caracterizada como menos
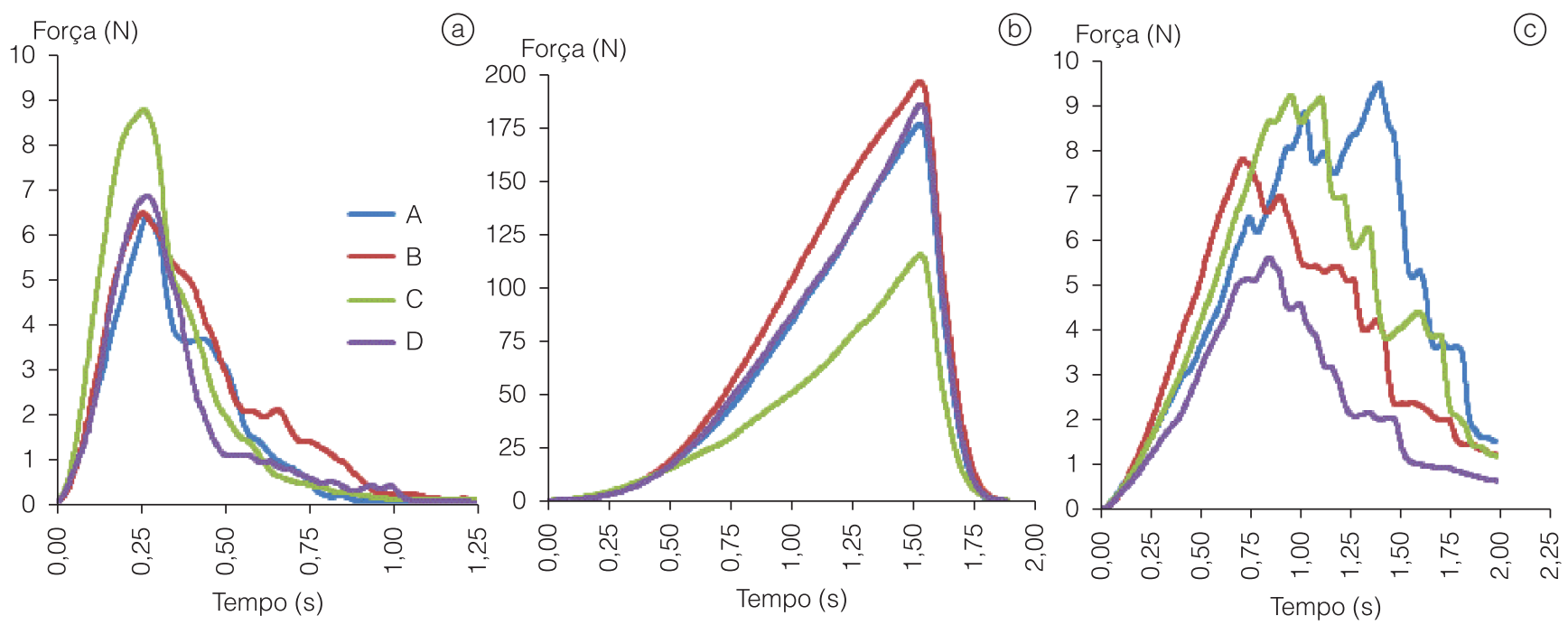

Figura 1. Perfil médio* representativo de penetração (a), compressão (b) e ruptura (c) dos biscoitos. *Cada curva média corresponde a dez repetições de cada teste.

Tabela 5. Caracterização instrumental de textura ${ }^{1}$ e $\operatorname{cor}^{2}$ dos biscoitos.

\begin{tabular}{|c|c|c|c|c|c|}
\hline Amostra & $\begin{array}{l}\text { Força de penetração } \\
\text { (N) }\end{array}$ & $\begin{array}{l}\text { Força de compressão } \\
\text { (N) }\end{array}$ & $\begin{array}{c}\text { Força de ruptura } \\
\text { (N) }\end{array}$ & $\begin{array}{l}\text { Luminosidade } \\
\left(L^{*}\right)\end{array}$ & $\begin{array}{c}\text { Tonalidade cromática } \\
\qquad\left(\mathbf{H}^{*}\right)\end{array}$ \\
\hline \multirow[t]{2}{*}{ A } & $8,9 \pm 1,7^{a}$ & $176,8 \pm 6,6^{a}$ & $15,2 \pm 3,2^{a}$ & $69,1 \pm 2,6^{b}$ & $74,0 \pm 1,8^{b}$ \\
\hline & (19) & (4) & (21) & (4) & (2) \\
\hline \multirow[t]{2}{*}{ B } & $8,5 \pm 2,0^{a}$ & $196,8 \pm 6,8^{a}$ & $11,1 \pm 2,4^{b}$ & $61,4 \pm 3,6^{c}$ & $69,6 \pm 2,4^{c}$ \\
\hline & (24) & (3) & (22) & (6) & (3) \\
\hline \multirow[t]{2}{*}{ C } & $9,1 \pm 1,1^{a}$ & $115,4 \pm 4,2^{b}$ & $14,4 \pm 3,4^{a}$ & $74,8 \pm 3,6^{a}$ & $83,9 \pm 3,6^{a}$ \\
\hline & (12) & (4) & (23) & (5) & (4) \\
\hline \multirow[t]{2}{*}{ D } & $7,7 \pm 1,2^{a}$ & $186,1 \pm 7,1^{a}$ & $7,7 \pm 1,5^{c}$ & $66,8 \pm 3,4^{b}$ & $74,8 \pm 2,8^{b}$ \\
\hline & $(16)$ & (4) & $(20)$ & $(5)$ & (4) \\
\hline
\end{tabular}

${ }^{1}$ Média de dez análises \pm desvio padrão. ${ }^{2}$ Média de nove análises \pm desvio padrão. Em parênteses o valor do coeficiente de variação (CV); letras diferentes na mesma coluna indicam diferenças significativas $(p \leq 0,05)$. 
crocante, por apresentar a menor força de ruptura. As amostras A e C foram consideradas as mais crocantes, sendo que a $C$ se diferenciou por ser a amostra menos dura, por apresentar menor força de compressão e mostrar-se mais clara e amarelada do que as demais. Diversamente, a amostra B caracterizou-se como a mais escura e avermelhada (Figura 1, Tabela 5).
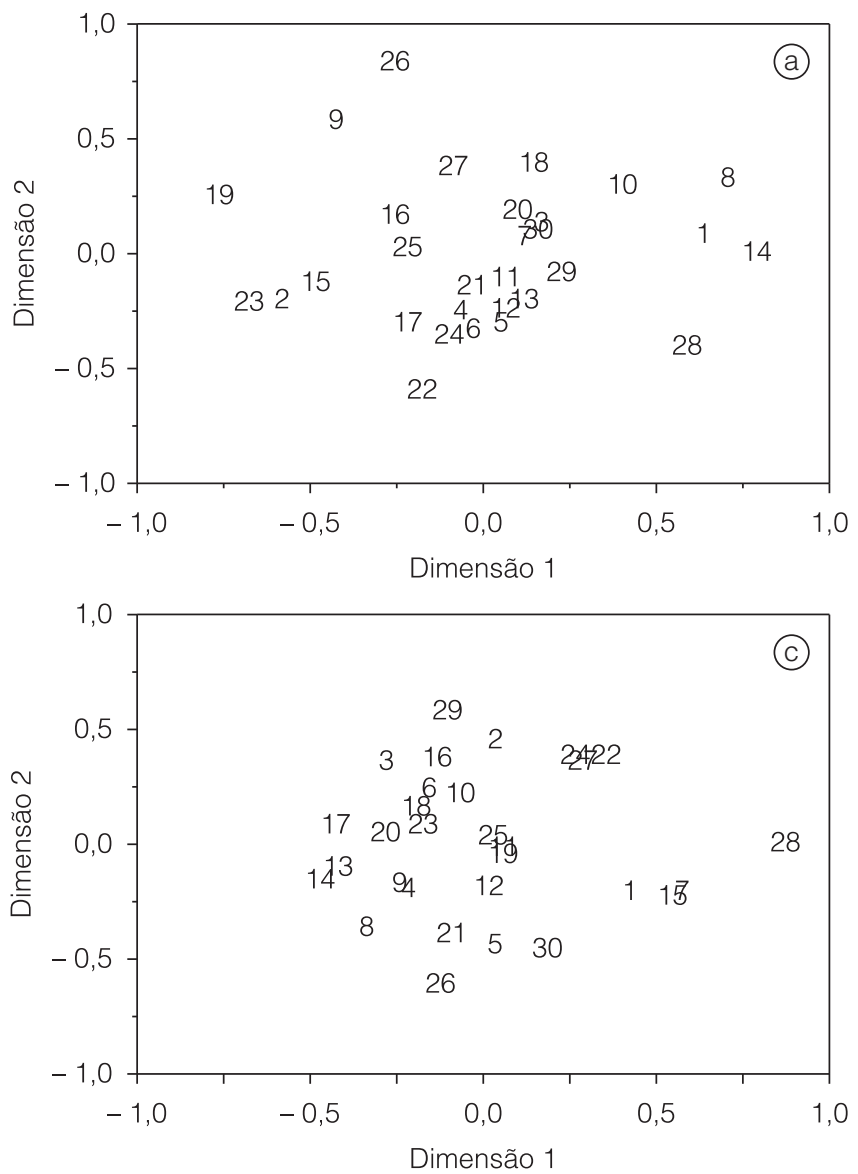

\subsection{Avaliação do desempenho dos julgadores}

A configuração geral dos julgadores, obtida da APG, mostrou comportamento semelhante e consensual para a equipe em ambas as técnicas (ADO e escala) (Figura 2). Foi observada baixa variância residual dos provadores, independentemente da técnica empregada (0,6\% para uso de escala e 0,5\% para ADO) (Figura 3).
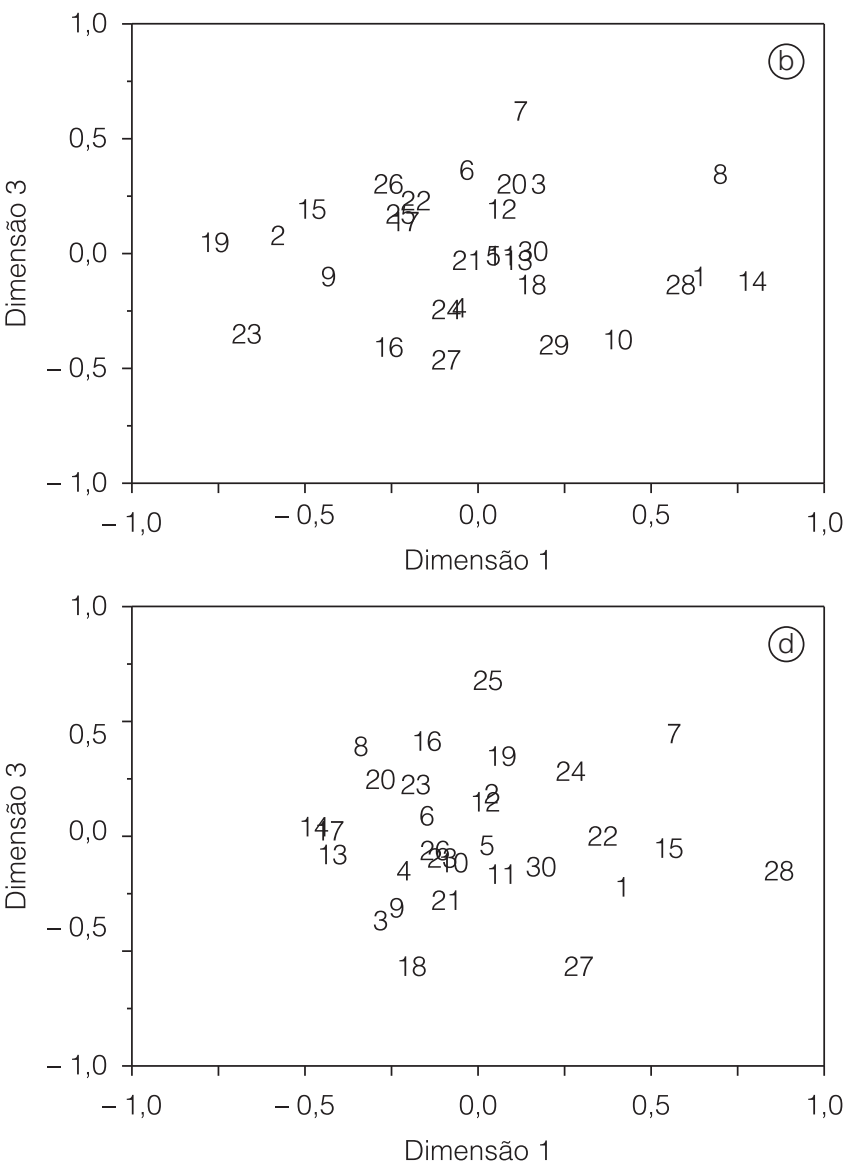

Figura 2. Configuração geral dos julgadores na ADO (a e b) e no uso de escala (c e d) gerada na APG para as dimensões 1 e 2 ( $a$ e c) e dimensões 1 e 3 (b e d), respectivamente.
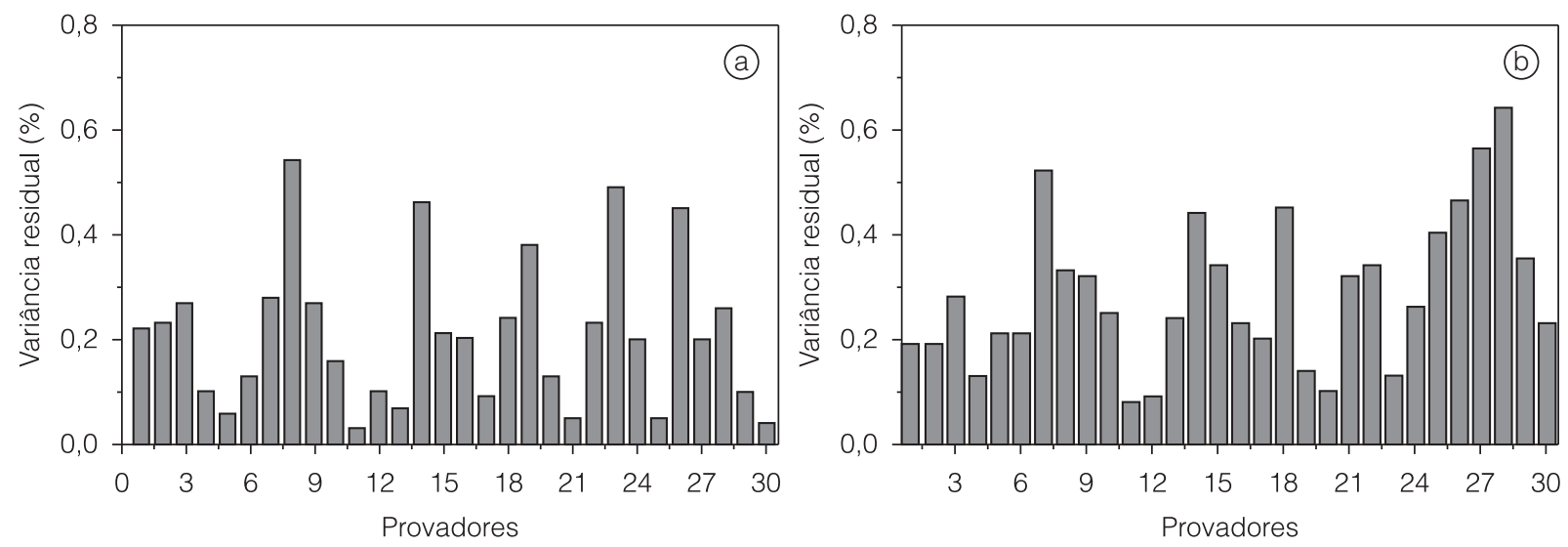

Figura 3. Distribuição da variância residual dos julgadores, gerada na APG, para ADO (a) e no emprego de escala (b). 
Carnelocce (2011), trabalhando com a ADO de refrescos, relatou boa concordância da equipe, mas variância residual mais alta $(1,0 \%)$, provavelmente em virtude da avaliação de um número maior de amostras (cinco bebidas) em três repetições de análise.

Richter et al. (2010), ao compararem ADO e análises descritivas tradicionais (ADQ e Perfil Livre) na avaliação de quatro pudins, verificaram que, mesmo após o treinamento quantitativo recebido pela equipe do $A D Q$, os julgadores apresentaram maior dispersão na configuração geral e variância residual de até 1,2\%. A equipe do Perfil Livre, que não recebeu treinamento do uso de escala, demonstrou um comportamento similar e variância residual de até $1,5 \%$. Os autores justificaram que a maior facilidade do procedimento de ordenação do que o da escala permitiu que a equipe da ADO apresentasse maior consenso e variância residual de até $0,5 \%$, similar à encontrada neste trabalho.

Os gráficos individuais de configuração das amostras de cada julgador (dados não mostrados) também apresentaram similaridade na discriminação dos produtos, quando comparados ao gráfico de consenso
(Figura 4), comprovando o consenso no comportamento da equipe e indicando a eficiência do treinamento.

É interessante observar que, neste trabalho, o treinamento qualitativo efetuado foi suficiente para que os julgadores não apresentassem divergência de comportamento, mesmo empregando um procedimento mais difícil (uso de escala). O procedimento de ordenação utilizado inicialmente pela equipe pode ter contribuído para maior consenso no uso posterior da escala. Kim e O'Mahoney (1998), em estudo sobre habilidade de discriminação de julgadores não treinados, relataram que os julgadores organizaram primeiro as amostras pela ordem de intensidade para depois distribuí-las na escala.

\subsection{Comparação entre a discriminação obtida pela ADO e pelo uso de escala}

As configurações de consenso das amostras obtidas pela APG foram avaliadas empregando-se solução tridimensional. Na ADO, observou-se que $94 \%$ da variabilidade dos dados foi explicada, em comparação a 91\% para uso de escala (Figura 4). Os atributos melhor correlacionados por dimensão para cada julgador estão indicados na Tabela 6.
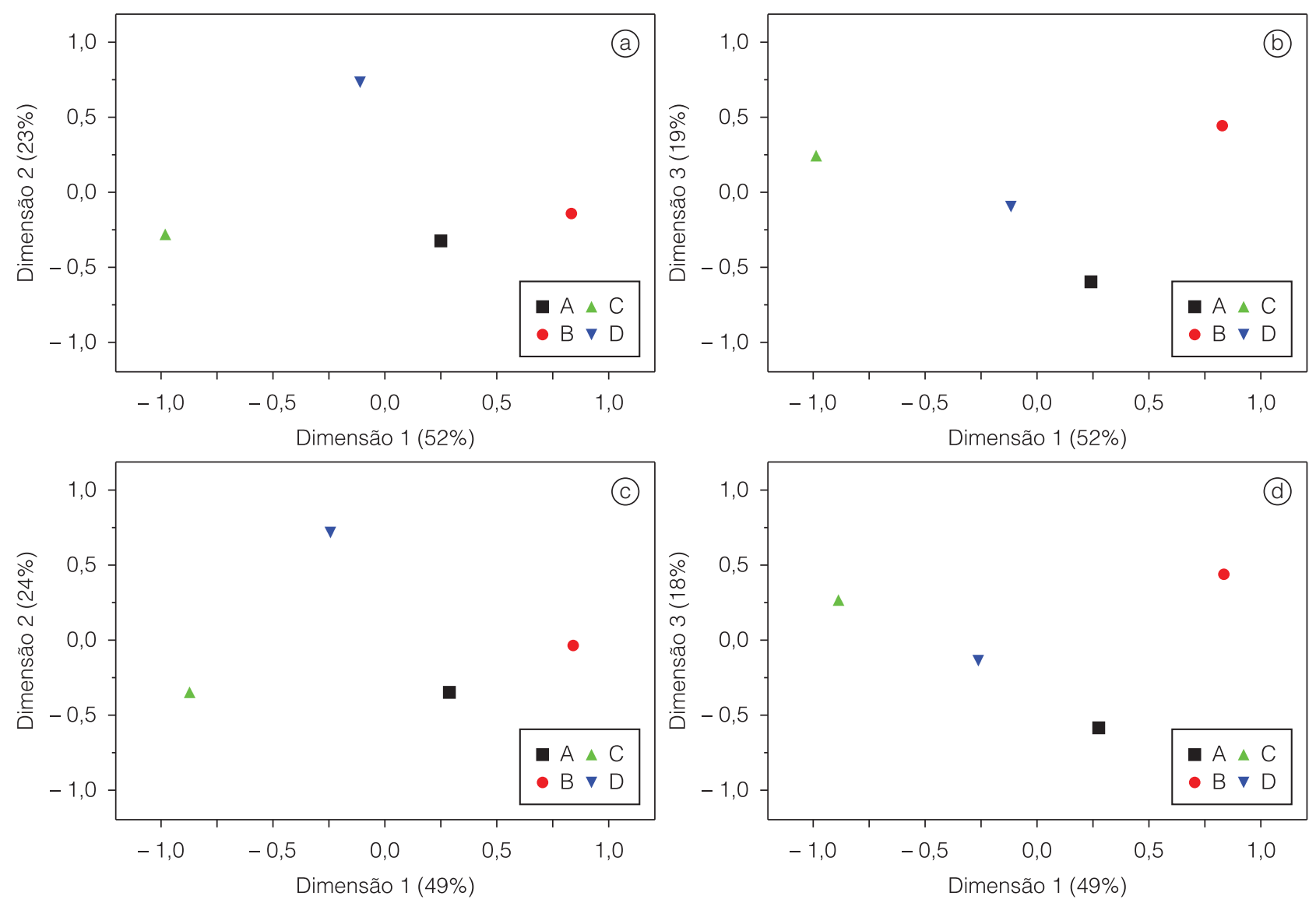

Figura 4. Configuração de consenso das amostras na ADO (a e b) e no uso de escala (c e d) gerada na APG para as dimensões 1 e 2 ( $a$ e c) e dimensões 1 e 3 ( $b$ e d), respectivamente. 


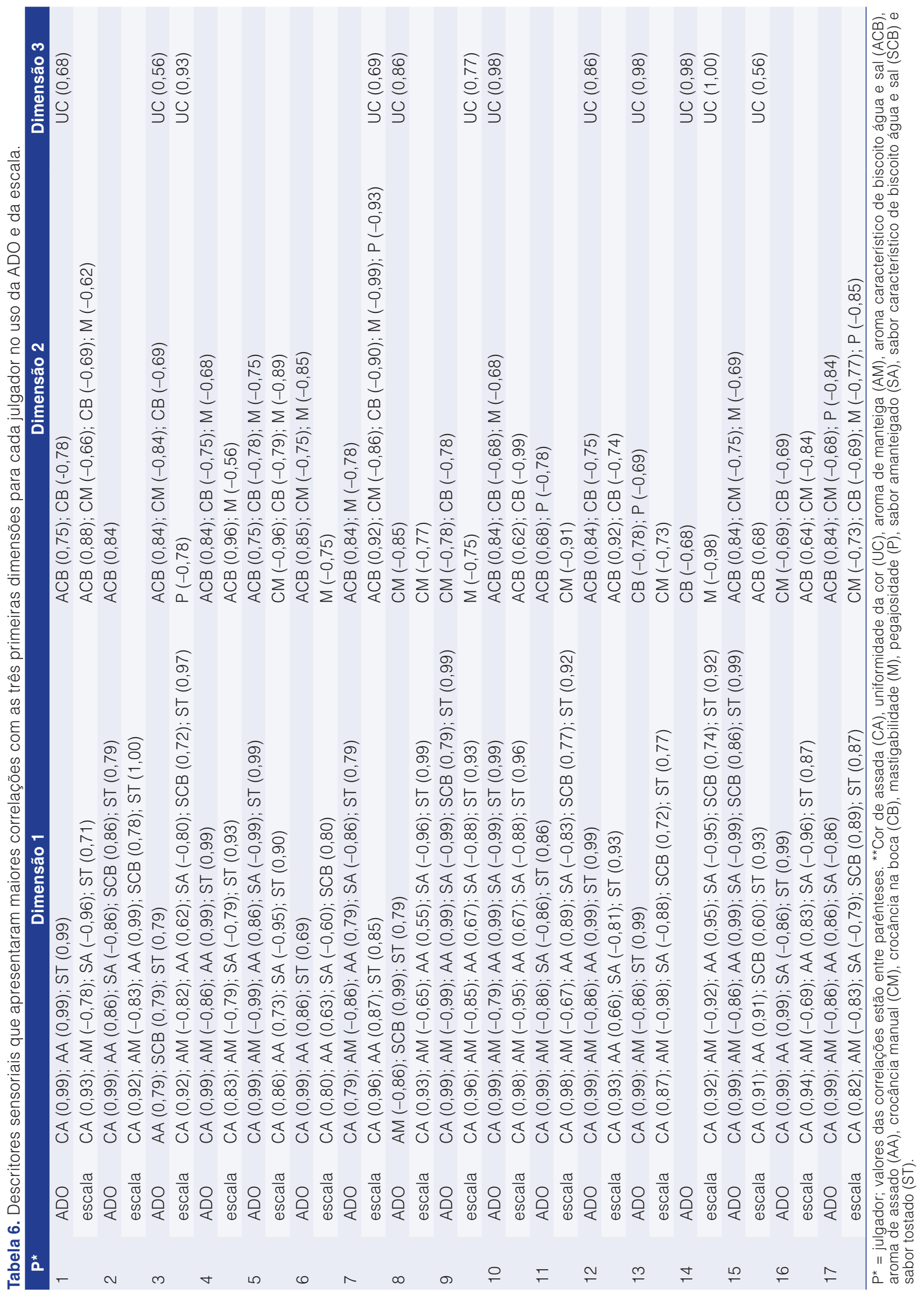




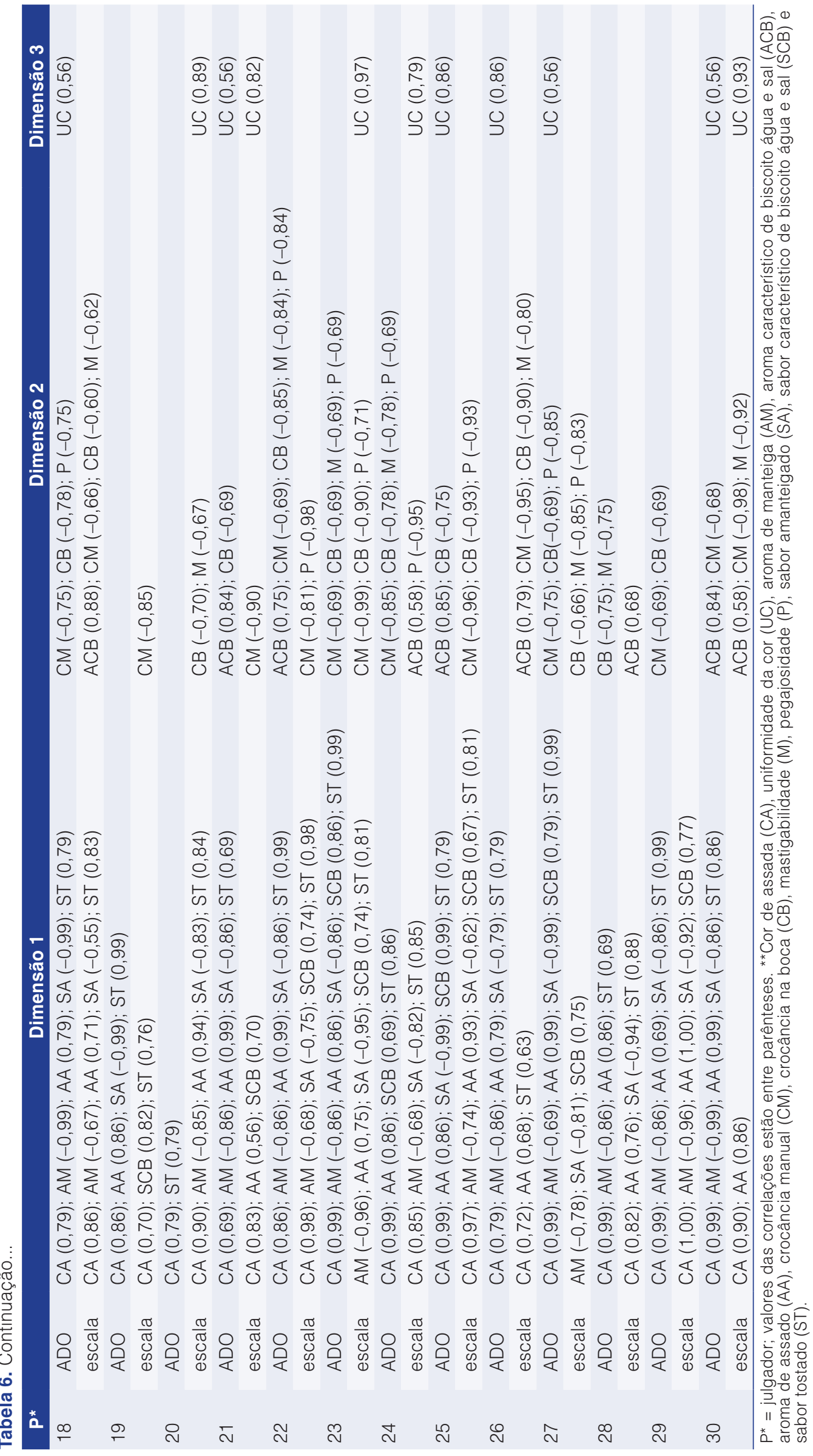


A dimensão 1 (D1) foi responsável por 52\% e 49\% de explicação para o emprego de ordenação e escala, respectivamente (Figura 4). Nas duas técnicas, D1 foi explicada, na direção positiva, pelos atributos: i) sabor tostado (alta correlação do atributo para 28 e 25 julgadores na ADO e na escala, respectivamente); ii) cor de assada (alta correlação para 27 e 28 julgadores na ADO e na escala); iii) aroma de assado (alta correlação para 25 e 22 julgadores na ADO e na escala), e iv) sabor característico de biscoito água e sal (alta correlação para nove e 15 julgadores na ADO e na escala). Na direção negativa, D1 foi explicada pelos atributos: aroma de manteiga (alta correlação para 20 e 20 julgadores na ADO e na escala) e sabor amanteigado (alta correlação para 19 e 23 julgadores na ADO e na escala) (Figura 4, Tabela 6). Essa dimensão contribuiu na discriminação de todas as amostras

A dimensão 2 (D2) foi responsável por 23\% e $24 \%$ de explicação para o emprego de ordenação e escala, respectivamente (Figura 4). Nas duas técnicas, D2 foi correlacionada com os atributos de: i) textura; ii) crocância na boca (alta correlação para 19 e 12 julgadores na ADO e na escala); iii) crocância manual (alta correlação para 14 e 16 julgadores na ADO e na escala); iv) mastigabilidade (alta correlação para dez e 13 julgadores na ADO e na escala), e v) pegajosidade (alta correlação para oito e oito, nas duas técnicas), na direção negativa. O aroma característico de biscoito água e sal (alta correlação para 16 e 12 julgadores na ADO e na escala), na direção positiva (Figuras 4a, c, Tabela 6), contribuiu principalmente na discriminação da amostra D.

A dimensão 3 (D3) foi responsável por 19\% e 28\% de explicação para o emprego de ordenação e escala, respectivamente (Figura 4). Nas duas técnicas, D3 foi caracterizada, basicamente, pelo atributo uniformidade da cor (alta correlação para 13 e dez julgadores na ADO e na escala) de forma positiva (Figuras 4b, d, Tabela 6), contribuindo para a discriminação entre as amostras $A$ e B.

A equipe apresentou altas correlações para os descritores de modo similar para os procedimentos de ordenação e escala. Observou-se um perfil concordante na caracterização e na discriminação das amostras estudadas, mostrando a equivalência do poder de discriminação dos métodos. Esse fato indica, também, que um treinamento qualitativo adequado e o uso de ordenação na classificação da intensidade dos atributos, precedendo a avaliação por escala, facilitaram o seu emprego e a obtenção de consenso da equipe. Pode-se, assim, observar que o procedimento de ordenação - ainda que imponha aos julgadores a obrigatoriedade de ordenar os biscoitos com relação a todos os atributos mesmo que não observem diferença em algum deles - permite caracterização e discriminação das amostras de forma similar ao obtido quando se usou uma escala.

\subsection{Caracterização dos biscoitos na ADO}

Para discriminação e descrição dos biscoitos estudados, foram utilizados os resultados da ADO, considerando-se os resultados do teste de Friedman (Tabela 7), a configuração de consenso (Figura 4) e correlações (Tabela 6), obtidos na APG.

$\mathrm{O}$ biscoito $\mathrm{C}$, alocado mais à esquerda no gráfico da configuração de consenso, caracterizou-se por ser menos assado, com menos cor e aroma de assado, e sabor de tostado, além de apresentar sabor e aroma de manteiga (Figura 4a, Tabelas 5-7).

Os biscoitos A e B, produzidos pelo mesmo fabricante, apresentaram características similares, diferenciando-se pelo atributo uniformidade da cor; o biscoito B, alocado na parte superior do diagrama, caracterizou-se por exibir cor mais homogênea do que o biscoito A (Figura 4b, Tabelas 6, 7). Na configuração de consenso, o biscoito $\mathrm{B}$, alocado mais à direita, foi descrito como mais assado/tostado, tanto na cor quanto no aroma e sabor, concordando com a análise instrumental de cor (Figura 4a e Tabela 5).

O biscoito $\mathrm{D}$, que havia sido descrito na análise instrumental como menos crocante (menor força de ruptura, Tabela 5), foi alocado na parte superior do gráfico, discriminado dos demais por ser menos crocante (Figura 4a)

Todas as amostras apresentaram em comum as características de serem pegajosas, possuírem

Tabela 7. Caracterização sensorial das amostras na $\mathrm{ADO}^{1,2}$

\begin{tabular}{lcccc}
\multicolumn{1}{c}{ Atributos } & A & Amostras \\
Cor de assada & $95^{\mathrm{a}}$ & $110^{\mathrm{a}}$ & $33^{\mathrm{c}}$ & $62^{\mathrm{b}}$ \\
Uniformidade da cor & $47^{\mathrm{b}}$ & $74^{\mathrm{a}}$ & $90^{\mathrm{a}}$ & $89^{\mathrm{a}}$ \\
Aroma de manteiga & $83^{\mathrm{b}}$ & $40^{\mathrm{c}}$ & $117^{\mathrm{a}}$ & $60^{\mathrm{bc}}$ \\
Aroma característico de & $76^{\mathrm{a}}$ & $89^{\mathrm{a}}$ & $35^{\mathrm{b}}$ & $100^{\mathrm{a}}$ \\
biscoito água e sal & & & & \\
Aroma de assado & $88^{\mathrm{ab}}$ & $106^{\mathrm{a}}$ & $33^{\mathrm{c}}$ & $73^{\mathrm{b}}$ \\
Crocância manual & $75^{\mathrm{ab}}$ & $80^{\mathrm{ab}}$ & $89^{\mathrm{a}}$ & $56^{\mathrm{b}}$ \\
Crocância na boca & $96^{\mathrm{a}}$ & $95^{\mathrm{a}}$ & $66^{\mathrm{b}}$ & $43^{\mathrm{b}}$ \\
Mastigabilidade & $77^{\mathrm{a}}$ & $87^{\mathrm{a}}$ & $73^{\mathrm{a}}$ & $63^{\mathrm{a}}$ \\
Pegajosidade & $81^{\mathrm{a}}$ & $67^{\mathrm{a}}$ & $80^{\mathrm{a}}$ & $72^{\mathrm{a}}$ \\
Sabor amanteigado & $73^{\mathrm{b}}$ & $45^{\mathrm{c}}$ & $114^{\mathrm{a}}$ & $68^{\mathrm{bc}}$ \\
Sabor característico de & $84^{\mathrm{a}}$ & $74^{\mathrm{a}}$ & $64^{\mathrm{a}}$ & $78^{\mathrm{a}}$ \\
biscoito água e sal & & & & \\
Sabor de tostado & $97^{\mathrm{a}}$ & $109^{\mathrm{a}}$ & $34^{\mathrm{c}}$ & $60^{\mathrm{b}}$
\end{tabular}

${ }^{1}$ Soma dos valores de Ordenação para 30 julgadores. ${ }^{2}$ Letras diferentes na mesma linha indicam diferença significativa $(p \leq 0,05)$, de acordo com o tabelado por Newell e MacFarlane (1987). Valor crítico: 26 (quatro amostras e 30 julgadores). 
Análise Descritiva por Ordenação: aplicação na caracterização sensorial de biscoitos laminados salgados CARNELOCCE, L. et al.

mastigabilidade e sabor característico de biscoito água e sal (Figura 4a, Tabelas 6, 7). A avaliação da força de penetração instrumental, que caracteriza de forma global a textura dos produtos (SALVADOR et al., 2009), já havia mostrado similaridade de textura entre as amostras (Tabela 5).

Desse modo, a equipe conseguiu caracterizar e discriminar as amostras, algo que pode ser comprovado pela conformidade com os teores de gordura e sódio (Tabela 1) e as análises instrumentais de textura e cor (Figura 1, Tabela 5) dos biscoitos.

Neste estudo, foi usado o mesmo número de amostras (quatro) proposto por Richter et al. (2010) no desenvolvimento do método, tendo em vista a limitação do procedimento de ordenação em si. Carnelocce (2011), apesar de obter boa performance da equipe de ADO, relatou dificuldade dos julgadores para ordenar cinco refrescos. Assim, pelo descrito na literatura e pelos resultados obtidos neste trabalho, preconiza-se para a ADO, principalmente no caso de matrizes mais complexas, o emprego de quatro amostras.

\section{Conclusões}

O método da Análise Descritiva por Ordenação permitiu a caracterização e a discriminação dos biscoitos de modo concordante com a composição indicada no rótulo e os dados instrumentais de textura e cor. A equipe discriminou e caracterizou as amostras de forma semelhante (similaridade na configuração de consenso e nos atributos), quando se comparou o procedimento de ordenação e o uso de escala. Cor e aroma de assado, sabor tostado, aroma de manteiga e sabor amanteigado, crocância (manual e na boca) e uniformidade da cor foram os atributos mais importantes na discriminação.

\section{Referências}

BARYLKO-PIKIELNA, N.; MATUSZEWSKA, I.; JERUSZKA, M.; KOZLOWSKA, K.; BRZOZOWSKA, A.; ROSZKOWSKI, W. Discriminability and appropriateness of category scaling versus ranking methods to study sensory preferences in elderly. Food Quality and Preference, Oxford, v. 15, n. 2, p. 167-175, 2004. http://dx.doi.org/10.1016/S0950-3293(03)00055-7

BOURNE, M. C. Texture profile analysis. Food Technology, Chicago, v. 32, p. 62-66, 1978.

CARNELOCCE, L. Análise Descritiva por Ordenação: Validação da Técnica e Aplicação a Diferentes Matrizes Alimentares. 2011. 86 f. Dissertação (Mestrado em Ciência de Alimentos)-Universidade Estadual de Londrina, Londrina, 2011.

CHAUVIN, M. A.; YOUNCE, F.; ROSS, C.; SWANSON, B. Standard scales for crispness, crackliness and crunchiness in dry and wet foods: relationship with acoustical determinations. Journal of
Texture Studies, Trumbull, v. 39, n. 4, p. 345-368, 2008. http:// dx.doi.org/10.1111/j.1745-4603.2008.00147.x

CHEN, J.; KARLSSON, C.; POVEY, M. Acoustic envelope detector for crispness assessment of biscuits. Journal of Texture Studies, Trumbull, v. 36, n. 2, p.139-156, 2005. http:// dx.doi.org/10.1111/j.1745-4603.2005.00008.x

DELARUE, J.; SIEFFERMANN, J. M. Sensory mapping using Flash Profile. Comparison with a conventional descriptive method for the evaluation of the flavour of fruit dairy products. Food Quality and Preference, Oxford, n.15, n. 4, p. 383-392, 2004. http://dx.doi.org/10.1016/S0950-3293(03)00085-5

DURÁN, L. Evaluación de la textura: correlación entre medidas sensoriales e instrumentales. In: ALMEIDA, T. C. A.; $\mathrm{HOUCH}$, G.; DAMÁSIO, M. H.; SILVA, M. A. A. P. (Eds.). Avanços em Análise Sensorial. São Paulo: Livraria Varela, 1999. p. 35-48.

INTERNATIONAL ORGANIZATION FOR STANDARDIZATION - ISO ISO 8597: Sensory Analysis. Methodology Ranking. Geneva: ISO copyright office, 2006. p. 26.

KIM, K.; O'MAHONY, M. A new approach to category scales of intensity: traditional versus rank-rating. Journal of Sensory Studies, Trumbull, v. 13, n. 3, p. 241-249, 1998. http://dx.doi. org/10.1111/j.1745-459X.1998.tb00086.x

LAGUNA, L.; SALVADOR, A.; SANZ, T.; FISZMAN, S. M. Performance of a resistant starch rich ingredient in the baking and eating quality of short-dough biscuits. LWT - Food Science and Technology, Oxford, v. 44, n. 3, p. 737-746, 2010. http:// dx.doi.org/10.1016/j.Iwt.2010.05.034

LEE, H. S.; O'MAHONY, M. Sensory evaluation and marketing: measurement of a consumer concept. Food Quality and Preference, Oxford, v. 16, n. 3, p. 227-235, 2005. http://dx.doi. org/10.1016/j.foodqual.2004.04.013

LIEM, D. G.; MARS, M.; GRAAF, C. Consistency of sensory testing with 4- and 5-year-old children. Food Quality and Preference, Oxford, v. 15, n. 6, p. 541-548, 2004. http://dx.doi. org/10.1016/j.foodqual.2003.11.006

MEILGAARD, M. C.; CIVILLE, G. V.; CARR, B. T. Sensory Evaluation Techniques. 3rd ed. Boca Raton: CRC Press, 1999. 387 p.

MOSKOWITZ, H. R. Product Testing and Sensory Evaluation of Foods - Marketing and R\&D Approaches. Westport: Food and Nutrition Press, Inc., 1983. 605 p.

NEWELL, G. J.; MacFARLANE, J. D. Expanded tables for multiple comparison procedures in the analysis of ranked data. Journal of Food Science, Chicago, v. 52, n. 6, p. 1721-1725, 1987. http:// dx.doi.org/10.1111/j.1365-2621.1987.tb05913.x

OP\&P PRODUCT RESEARCH. Senstools Versão 2.3. Utrecht: OP\&P Product Research, 1995-1998. Conjunto de Programas 1CD-ROOM. 
Análise Descritiva por Ordenação: aplicação na caracterização sensorial de biscoitos laminados salgados CARNELOCCE, L. et al.

RICHTER, V. B.; AVANCINI, T. C.; PRUDENCIO, S. H.; BENASSI, M. T. Proposing a ranking descriptive sensory method. Food Quality and Preference, Oxford, v. 21, n. 6, p. 611-620, 2010. http://dx.doi.org/10.1016/j.foodqual.2010.03.011

RODRIGUE, N.; GUILLET, M.; FORTIN, J.; MARTIN, J. F. Comparing information obtained from ranking and descriptive tests of four sweet corn products. Food Quality and Preference, Oxford, v. 11, n. 1-2, p. 47-54, 2000. http://dx.doi.org/10.1016/ S0950-3293(99)00063-4

SALVADOR, A.; VARELA, P.; SANZ, T.; FISZMAN, S.M. Understanding potato chips crispy texture by simultaneous fracture and acoustic measurements, and sensory analysis. LWT - Food Science and Technology, Oxford, v. 42, n. 3, p. 763-767, 2009. http://dx.doi.org/10.1016/j.Iwt.2008.09.016
SCHIFFERSTEIN, H. N. J. The perceived importance of sensory modalities in product usage: A study of self-reports. Acta Psychologica, Oxford, v. 121, n. 1, p. 41-64, 2006. http://dx.doi. org/10.1016/j.actpsy.2005.06.004

STATSOFT. STATISTICA for Windows. Computer program manual. versão 7.1 Tulsa: Statsoft Inc., 2001.

STONE, H.; SIDEL, J. L. Quantitative descriptive analysis: developments, applications and the future. Food Technology, Chicago, v. 52, n. 8, p. 48-52, 1998.

VARELA, P.; SALVADOR, A.; FISZMAN, S. On the assessment of fracture in brittle foods II. Biting or chewing?. Food Research International, Oxford, v. 42, n. 10, p. 1468-1474, 2009. http:// dx.doi.org/10.1016/j.foodres.2009.08.004 